University of Nebraska - Lincoln

DigitalCommons@University of Nebraska - Lincoln

Faculty Publications, Department of Physics and Astronomy

Research Papers in Physics and Astronomy

10-15-1997

\title{
Controlled formation of weakly bound atomic negative ions by electron transfer from state-selected Rydberg atoms
}

\author{
M. Reicherts \\ Fachbereich Physik der Universität - Kaiserslautern, Germany \\ T. Roth \\ Fachbereich Physik der Universität - D-67653 Kaiserslautern, Germany \\ A. Gopalan \\ Fachbereich Physik der Universität - D-67653 Kaiserslautern, Germany \\ M.-W. Ruf \\ Fachbereich Physik der Universität - D-67653 Kaiserslautern, Germany \\ Harmut Hotop \\ Universität Kaiserslautern, hotop@physik.uni-kl.de \\ See next page for additional authors
}

Follow this and additional works at: https://digitalcommons.unl.edu/physicsfacpub

Part of the Physics Commons

Reicherts, M.; Roth, T.; Gopalan, A.; Ruf, M.-W.; Hotop, Harmut; Desfrançuois, C.; and Fabrikant, llya I., "Controlled formation of weakly bound atomic negative ions by electron transfer from state-selected Rydberg atoms" (1997). Faculty Publications, Department of Physics and Astronomy. 65.

https://digitalcommons.unl.edu/physicsfacpub/65

This Article is brought to you for free and open access by the Research Papers in Physics and Astronomy at DigitalCommons@University of Nebraska - Lincoln. It has been accepted for inclusion in Faculty Publications, Department of Physics and Astronomy by an authorized administrator of DigitalCommons@University of Nebraska Lincoln. 


\section{Authors}

M. Reicherts, T. Roth, A. Gopalan, M.-W. Ruf, Harmut Hotop, C. Desfrançuois, and llya I. Fabrikant 
Europhys. Lett., 40 (2), pp. 129-134 (1997)

\title{
Controlled formation of weakly bound atomic negative ions by electron transfer from state-selected Rydberg atoms
}

\author{
M. Reicherts ${ }^{1}$, T. Roth ${ }^{1}$, A. Gopalan ${ }^{1}$, M.-W. RuF $^{1}$ \\ H. Hotop ${ }^{1}$, C. DesfrançOIS ${ }^{2}$ and I. I. FAbrikant ${ }^{3}$ \\ ${ }^{1}$ Fachbereich Physik der Universität - D-67653 Kaiserslautern, Germany \\ 2 Laboratoire de Physique des Lasers, Université Paris-Nord \\ F-93430 Villetaneuse, France \\ 3 Department of Physics and Astronomy, University of Nebraska \\ Lincoln, Nebraska 68588-0111, USA
}

(received 12 August 1997; accepted 4 September 1997)

PACS. 34.70+e - Charge transfer.

PACS. $32.10 \mathrm{Hq}$ - Ionization potentials, electron affinities.

PACS. 41.75Cn - Negative-ion beams.

\begin{abstract}
Using crossed atomic beams and mass spectrometric ion detection we have studied the formation of negative $\mathrm{Ca}$ ions in slow collisions between laser-excited state-selected $\mathrm{Ne}^{* *}(n s, J=2)$ and $\mathrm{Ne}^{* *}(n d, J=4)$ Rydberg atoms and ground state Ca atoms. The rate coefficients exhibit resonant behaviour with maxima at an effective principal quantum number $n^{*}$ of about 11.5 and an estimated peak value of about $10^{-8} \mathrm{~cm}^{3} \mathrm{~s}^{-1}$. Two different theoretical calculations, based on a curve-crossing model and on a modified adiabatic theory, respectively, yield $n^{*}$-dependences of the rate coefficients in good agreement with the experimental results.
\end{abstract}

The negative ions of $\mathrm{Ca}, \mathrm{Sr}$, and $\mathrm{Ba}$ have received a lot of attention over the last ten years [1]-[8] as prototype examples of weakly bound systems whose stability is determined by intricate electron correlation effects. Precise experimental values of their binding energies have only very recently become available through a combination of laser photodetachment with resonance ionization spectroscopy [6]-[8]. For $\mathrm{Ca}^{-}$, for example, the binding energies were determined as 24.55(10) $\mathrm{meV}$ for $\mathrm{Ca}^{-}\left({ }^{2} P_{1 / 2}\right)$ and $19.73(10) \mathrm{meV}$ for $\mathrm{Ca}^{-}\left({ }^{2} P_{3 / 2}\right)$ [7]. In most experiments these weakly bound atomic negative ions are produced by passage of the respective positive ions at keV energies through an appropriate gaseous target [1]. Recently, McLaughlin and Duquette [9] indicated an alternative route towards the production of $\mathrm{Ca}^{-}$ions: studying ion formation in $\mathrm{Ca}^{* *}(n d)+\mathrm{Ca}$ collisions at thermal energies, they reported a sharp peak in both $\mathrm{Ca}^{+}$and $\mathrm{Ca}^{-}$production at $n=25$, corresponding to an effective principal quantum number of $n^{*}=23.9$. Using a generalization of the quasi-free-electron model, Fabrikant [10] interpreted these results in terms of a resonant electron transfer process in which the Rydberg binding energy $E_{0} / 2 n^{* 2}\left(E_{0}=27.21 \mathrm{eV}\right)$ essentially matches the electron affinity for Ca; the experimental peak location $\left(n^{*}=23.9\right)$ suggested $\mathrm{EA}(\mathrm{Ca})=24(1) \mathrm{meV}$ in contrast to the then

(C) Les Editions de Physique 

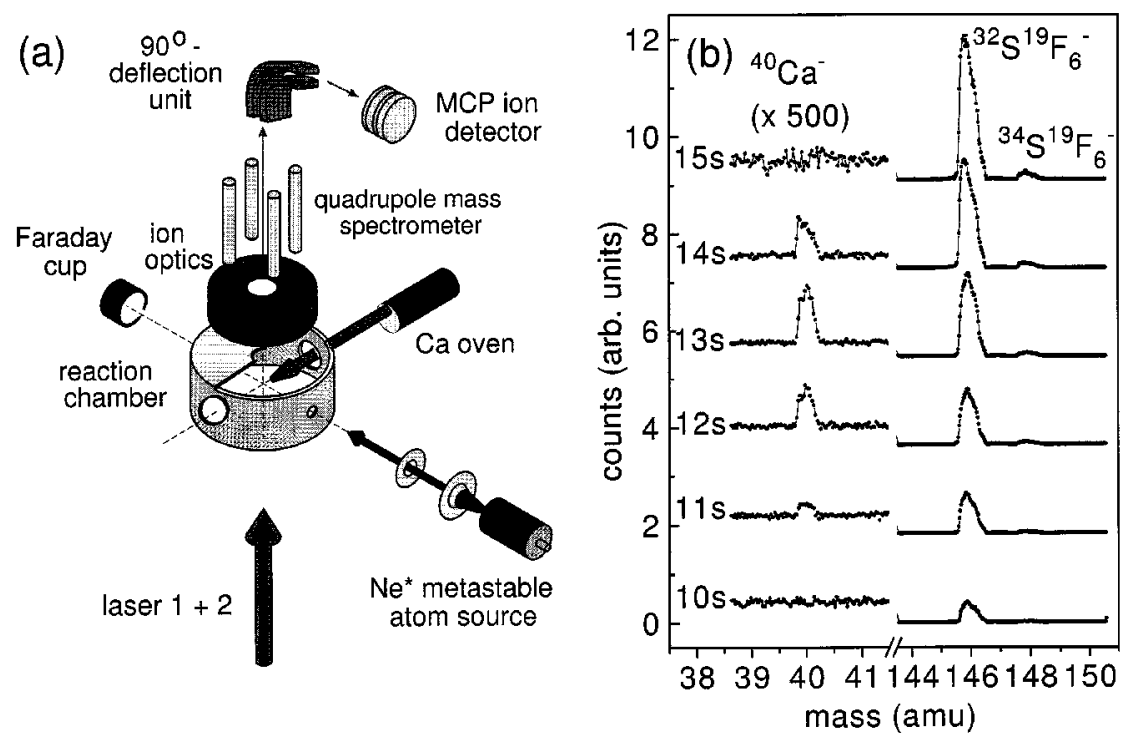

Fig. 1. - (a) Experimental setup for mass spectrometric studies of $\mathrm{Ca}^{-}$formation in thermal energy collisions of laser-excited $\mathrm{Ne}^{* *}(n s, n d)$ Rydberg atoms with $\mathrm{Ca}$ atoms. (b) $n$-dependent negative ion mass spectra resulting from collisions of state-selected $\mathrm{Ne}^{* *}(n s)$ Rydberg atoms with Ca atoms. For clarity, the zero intensity line of the consecutive mass spectra has been shifted.

accepted value $\mathrm{EA}(\mathrm{Ca})=18.4(25) \mathrm{meV}[1]$. In this context the recent studies of Desfrançois et al. [11]-[13] on the controlled production of dipole-bound molecular negative ions $\mathrm{XY}^{-}$with binding energies in the range from $20 \mathrm{meV}$ to below $1 \mathrm{meV}$ are of great interest. Using electron transfer from state-selected $\mathrm{Xe}^{* *}(n f)$ Rydberg atoms, they found resonant behaviour of the $n$-dependent rate coefficients $k_{n \ell}=\left\langle\sigma_{n \ell} v_{\text {rel }}\right\rangle$ (i.e. the product of the cross section with the collision velocity averaged over the experimental velocity distributions) for $\mathrm{XY}^{-}$formation. From their data they determined a simple empirical relation between the binding energy EA of the $\mathrm{XY}^{-}$ion and the value of the (effective) principal quantum number $n_{\max }^{*}$ for which the cross section attains its maximum, namely $n_{\max }^{*}=(23 \mathrm{eV} / \mathrm{EA})^{(1 / 2.8)}[11],[13]$. For an electron affinity EA of $20 \mathrm{meV}$, for example, the maximum is expected to occur at $n_{\max }^{*}=12.4$ in obvious contrast to the findings of McLaughlin and Duquette [9]. Lorensen et al. [14] have recently demonstrated that $\mathrm{Ca}^{+}$formation following excitation of $\mathrm{Ca}^{* *}(25 d)$ with the scheme used in [9] is in fact due to a resonant atomic three-photon ionization process and therefore not the result of $\mathrm{Ca}^{* *}(25 d)+\mathrm{Ca}$ collisions. In the meantime Khrebtukov and Fabrikant [15] developed an improved theoretical model for the description of negative ion formation through Rydberg electron transfer. Using $\mathrm{EA}(\mathrm{Ca})=18.4 \mathrm{meV}$ and the experimental conditions of the single-beam experiment in [9], they predicted resonant behaviour of the $\mathrm{Ca}^{-}$production cross section with a maximum at about $n^{*}=10.2$ (fig. 6 in [15]). In this letter we report new experimental results for $\mathrm{Ca}^{-}$formation in collisions between ground state $\mathrm{Ca}$ atoms and state-selected $\mathrm{Ne}^{* *}(n s)$ and $\mathrm{Ne}^{* *}(n d)$ Rydberg atoms. We find resonant behaviour of the rate coefficients with a peak at an effective principal quantum number of about $n^{*}=11.5$. For comparison and interpretation we have carried out calculations which are based on two different theoretical approaches [13], [15].

In our experiment (fig. 1 (a)) a collimated metastable $\mathrm{Ne}^{*}\left(3 s^{3} P_{2,0}\right)$ atom beam, originating from a differentially pumped dc discharge source, is crossed by a collimated Ca beam produced 
under effusive conditions in a differentially pumped, resistively heated oven. The $\mathrm{Ne}^{*}$ beam has supersonic character with an average velocity of about $930 \mathrm{~m} / \mathrm{s}$ and a velocity width of about 35\% (FWHM). The Ca beam is Maxwellian with a temperature of about $1100 \mathrm{~K}$. In the crossing region of the two beams their densities amount to about $2 \times 10^{6} \mathrm{~cm}^{-3}\left(\mathrm{Ne}^{*}\right)$ and $6 \times 10^{9} \mathrm{~cm}^{-3}(\mathrm{Ca}) . \mathrm{Ne}^{* *}\left(n s, J=2\right.$; quantum defect $\left.\mu_{s}=1.32[16]\right)$ or $\mathrm{Ne}^{* *}$ (nd, $J=4$; $\mu_{d}=0.02$ [16]) Rydberg states are produced in a controlled way by two-step transverse laser excitation of the $\mathrm{Ne}^{*}\left({ }^{3} P_{2}\right)$ atoms via the intermediate $\mathrm{Ne}^{*}\left(3 p{ }^{3} D_{3}\right)$ level [16], [17]; a single-mode ring dye laser, which is locked to the atomic transition $(640.4 \mathrm{~nm})$ by polarization spectroscopy in an rf discharge, is used for the first step $(640.4 \mathrm{~nm})$ and a stabilized single mode dye laser (Stilben 3, 420-460 nm) for the second transition. Mass spectra of positive ions (resulting from Penning ionization, PI) and of negative ions due to the reactions $\mathrm{Ne}^{* *}(n \ell)+$ $\mathrm{Ca} \rightarrow \mathrm{Ne}^{+}+\mathrm{Ca}^{-}$are measured with a quadrupole mass spectrometer (Balzers), equipped with a dual channel plate detector. For calibration purposes $\mathrm{SF}_{6}$ gas is injected into the reaction region through a conical nozzle surrounding the $\mathrm{Ca}$ beam; the $\mathrm{SF}_{6}$ density amounted to about $2 \times 10^{11} \mathrm{~cm}^{-3}$. The $n$-dependent rate coefficients for $\mathrm{SF}_{6}^{-}$formation in the electron transfer reactions $\mathrm{Ne}^{* *}(n s, n d)+\mathrm{SF}_{6} \rightarrow \mathrm{Ne}^{+}+\mathrm{SF}_{6}^{-}$are rising towards higher $n$ in the range of interest and are known from previous work [17], [18]. With this knowledge we determine relative rate coefficients for $\mathrm{Ca}^{-}$production from the $n$-dependent, mass-analyzed signals for $\mathrm{Ca}^{-}$and $\mathrm{SF}_{6}^{-}$shown for $\mathrm{Ne}^{* *}(n s)$ collisions in fig. 1 (b). We note that the $\mathrm{SF}_{6}^{-}$intensities in fig. 1 (b) have been normalized such that they mirror the $n$-dependence of the known rate coefficients [17]. Therefore the $\mathrm{Ca}^{-}$intensities reflect in a direct way the respective variation of the rate coefficient which exhibits resonant behaviour with a maximum at $n=13$ $\left(n^{*}=11.68\right)$. Analogous data have been obtained for $\mathrm{Ne}^{* *}(n d)$ collisions; the $n^{*}$-dependence is very similar with the maximum occurring at about $n^{*}=11.5$. Figure 2 summarizes the experimental data and compares them with the results of theoretical calculations to be discussed below. Our results are at variance with the experiments of McLaughlin and Duquette [9]. We find the maximum rate coefficient — consistently for $\mathrm{Ne}^{* *}(n s)$ and $\mathrm{Ne}^{* *}(n d)$ electron transfer - at an effective principal quantum number of about 11.5 which is quite close to the value expected from the empirical formula due to Desfrançois et al. [11], [13]. The ratio of the peak rate coefficients is determined as $k_{13 s} / k_{12 d}=1.5$. Using the experimentally known $\mathrm{Ca}$ and $\mathrm{SF}_{6}$ densities in conjunction with the measured value for $\mathrm{SF}_{6}^{-}$formation of $k_{13 s}=8.6 \times 10^{-8} \mathrm{~cm}^{3} \mathrm{~s}^{-1}( \pm 50 \%)$ [17] and assuming equal overall detection efficiencies for $\mathrm{Ca}^{-}$and $\mathrm{SF}_{6}^{-}$ions, we estimate the absolute value of the $13 \mathrm{~s}$ rate coefficient for $\mathrm{Ca}^{-}$formation as $k_{13 s}=1 \times 10^{-8} \mathrm{~cm}^{3} \mathrm{~s}^{-1}$ (to within a factor of three).

For comparison with our experimental results we have carried out calculations of the $\mathrm{Ca}^{-}$production cross sections as a function of $n^{*}$ and of collision velocity $v_{\text {rel }}$ using i) a parameter-free curve crossing model [13] and ii) an extended version of a modified adiabatic theory [15] (atomic units are used in the following expressions). In model i) the cross section for negative ion formation is given by $\sigma_{n \ell}\left(v_{\text {rel }}\right)=2 \pi \int_{0}^{R_{\mathrm{c}}} P(b) b \mathrm{~d} b$, where $R_{\mathrm{c}}=\left(1 / 2 n^{* 2}-\right.$ $\mathrm{EA})^{-1}$ is the crossing radius between the initial (flat) covalent and the ionic curve and $P(b)$ represents the impact parameter dependent probability for ion pair formation. In the two-channel case we simply have $P(b)=2 p_{c i}\left(1-p_{c i}\right)$ with the Landau-Zener probability $p_{c i}$ for switching from the covalent channel $(c)$ to the final ionic channel $(i)$ given by $p_{c i}=$ $1-\exp \left[-2 \pi H_{c i}\left(R_{\mathrm{c}}\right)^{2} R_{\mathrm{c}}^{2} / v_{\mathrm{r}}\left(R_{\mathrm{c}}, b\right)\right]$, where $v_{\mathrm{r}}$ denotes the radial velocity and $H_{c i}$ is the exchange coupling element which is given by $H_{c i}\left(R_{\mathrm{c}}\right)=\left(N_{i} / 2\right) f_{c i} X_{n \ell}\left(R_{\mathrm{c}}\right)$ [13], [19]. $N_{i}$ represents the normalization constant of the electron wave function in the negative ion (for the definition of $N_{i}$ see [13], [19]). $X_{n \ell}\left(R_{\mathrm{c}}\right)$ is the value of the unperturbed radial wave function of the Rydberg electron at $r=R_{\mathrm{c}}$. The factor $f_{c i}$ depends on the orbital angular momenta $\ell, \ell_{i}$ and their common projection $\left(m=m_{i}\right)$ on the internuclear axis (for $\mathrm{Ca}^{-}, \ell_{i}=1, m_{i}=0,1$ ), 

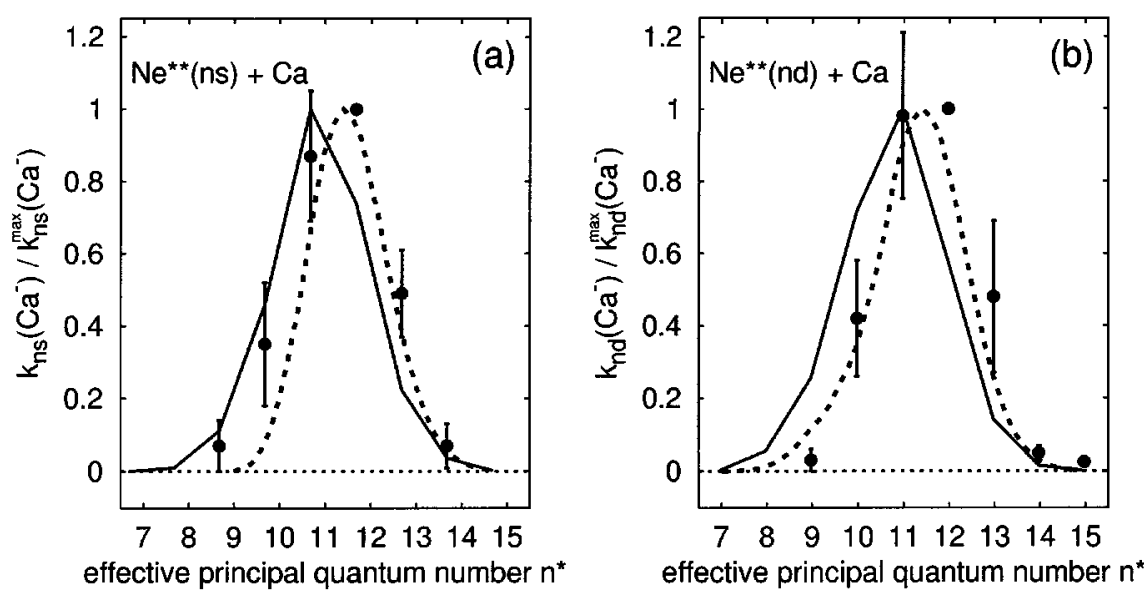

Fig. 2. - Comparison of experimental and theoretical results for the relative $n$-dependent rate coefficients $k_{n \ell}\left(\mathrm{Ca}^{-}\right)$for $\mathrm{Ca}^{-}$formation in thermal energy collisions of state-selected $\mathrm{Ne}^{* *}(n \ell)$ Rydberg atoms with $\mathrm{Ca}$ atoms: (a) $\mathrm{Ne}^{* *}(n s)+\mathrm{Ca}$. (b) $\mathrm{Ne}^{* *}(n d)+\mathrm{Ca}$. Solid lines: curve crossing model. Dashed lines: modified adiabatic theory. Filled circles with error bars: experimental data (average of three data runs in both cases).

i.e. $f_{c i}=(3(2 \ell+1))^{1 / 2}$ for $m=0, f_{c i}=(6 \ell(\ell+1)(2 \ell+1))^{1 / 2} /\left(2 R_{c}(2 \mathrm{EA})^{1 / 2}\right)$ for $m=1$, and $f_{c i}=0$ for $m>1$ [13], [19]. Realistic $\mathrm{Ca}^{-}$wave functions were calculated on the basis of a model potential with appropriate long-range behaviour $1 / r^{2}-\alpha / 2 r^{4}(\alpha=$ Ca polarizability $=159 \mathrm{au}[20]$ ) and was chosen to yield the proper Ca electron affinity; somewhat dependent on the choice of the potential we found $N_{i}=0.117$ for $\mathrm{Ca}^{-}\left({ }^{2} P_{3 / 2}\right)$ and $N_{i}=0.132$ for $\mathrm{Ca}^{-}\left({ }^{2} P_{1 / 2}\right)$ (within 5\%). We assume that the $\mathrm{Ne}^{+}\left({ }^{2} P_{3 / 2}\right)$ core acts as spectator and can be considered as fixed in the electron transfer process. The effects of multiple crossings associated with $n \ell$ Rydberg levels other than that of the entrance channel were included as described in [13]. Separate calculations with only one ionic channel $\mathrm{Ne}^{+}\left({ }^{2} P_{3 / 2}\right)+\mathrm{Ca}^{-}\left({ }^{2} P_{J}\right)$, using the appropriate electron affinities for $J=1 / 2$ or $J=3 / 2$, showed similar $n$-dependences with maxima at $n^{*}$ which were lower for $J=1 / 2$ by about one unit. Both final ionic channels were taken into account in a coupled channel calculation ignoring all interference effects. Carrying out the average over the experimental collision velocity distribution we obtain rate coefficients for $\mathrm{Ca}^{-}$formation in $\mathrm{Ne}^{* *}(n \ell)+\mathrm{Ca}$ collisions as shown by the solid lines in fig. 2. They reproduce the $n^{*}$-dependence of the experimental data in a satisfactory way; the predicted peak position is located at a somewhat smaller $n^{*}$ than the experimental value. The calculated peak rate coefficients of $k_{13 s}=1.7 \times 10^{-8} \mathrm{~cm}^{3} \mathrm{~s}^{-1}$ and $k_{12 d}=0.82 \times 10^{-8} \mathrm{~cm}^{3} \mathrm{~s}^{-1}$ agree with the estimated experimental values.

We also carried out calculations using a new version of the modified adiabatic theory which differs from that in [15] in two aspects. First, it incorporates the polarization attraction in the $\mathrm{e}^{-}+$Ca system. Second, it takes into account the decay of the formed negative ion due to the Coulomb field of the positive ion in a form proposed by Radtsig and Smirnov [21]. For the decay width we use theoretical results [22] which take into account the polarization potential of Ca. This decay is equivalent to the multiple crossing effect mentioned above where intersection with each sublevel of the degenerate Coulomb manifolds leads to the loss of flux from the ionic channel. To account for the $\mathrm{Ca}^{-}$fine structure two calculations were carried out with the appropriate electron affinities, and the resulting cross sections were averaged 
according to the statistical weights of the $\mathrm{Ca}^{-}$states. Our modified adiabatic theory yields $n^{*}$ dependences (dashed lines in fig. 2) which show good overall agreement with the experimental results; the absolute values of the calculated rate coefficients $\left(k_{13 s}=0.90 \times 10^{-8} \mathrm{~cm}^{3} \mathrm{~s}^{-1}\right.$, $\left.k_{12 d}=0.71 \times 10^{-8} \mathrm{~cm}^{3} \mathrm{~s}^{-1}\right)$ agree with those of the curve crossing model to within $50 \%$ and with the experimental estimates within the uncertainties of the latter. We note that in all the calculations it is assumed that the ionic complex $\mathrm{Ne}^{+}+\mathrm{Ca}^{-}$is not lost to the autoionization continuum (formation of $\mathrm{Ne}+\mathrm{Ca}^{+}+\mathrm{e}^{-}$in a Penning ionization type process) to which it is expected to be strongly coupled at short internuclear distances $R<10 a_{0}$ [20]. Estimates of the fraction of those collisions which reach such small distances show that loss due to autoionization should be less than about $10 \%$ and, therefore, only a small effect. From an experimental point of view, $\mathrm{Ca}^{-}$ions are subject to losses caused by blackbody-radiationinduced photodetachment [23] over the time interval from their creation to detection. In our experiment this interval amounts to about $76 \mu \mathrm{s}$, corresponding to photodetachment probabilities of about $15 \%$.

We conclude that we have observed resonant behaviour of the $n^{*}$-dependent rate coefficients measured for $\mathrm{Ca}^{-}$production in slow collisions of state-selected $\mathrm{Ne}^{*}$ Rydberg states with $\mathrm{Ca}$ atoms. The $n^{*}$-dependence is well recovered in two different calculations with a parameter-free curve crossing model and a modified adiabatic theory, respectively. The former has previously been found adequate for the description of Rydberg electron transfer to dipole-bound molecular negative ions [11]-[13]. We note that the situation for electron transfer from a Coulombic potential (i.e. from a Rydberg orbit) into a dipole-bound orbital (such as in $\mathrm{CH}_{3} \mathrm{CN}^{-}$) is quite different from that for formation of a weakly bound atomic negative ion such as $\mathrm{Ca}^{-}$. For the former the effective potential for the active electron along the line connecting the Rydberg core and the target dipolar molecule exhibits a broad maximum between the nuclear centers, with an energy close to the Rydberg binding energy when the system is close to the crossing radius for the entrance channel with $n_{\max }^{*}$ [11]. In contrast $\mathrm{Ca}^{-}\left({ }^{2} P_{J}\right)$ formation involves penetration of the centrifugal barrier associated with the $\ell_{i}=1$ symmetry (with our model potential we find a barrier height of about $0.1 \mathrm{eV}$ at an electron-Ca distance of about $11.6 a_{0}$ ). This difference between the two cases is reflected in the respective size of the normalization factor of the radial wave function of the weakly bound electron. Correspondingly, the peak of the $n$-dependent rate coefficient for $\mathrm{Ca}^{-}$formation occurs at a somewhat smaller value of the effective principal quantum number.

In future studies we plan to carry out experiments at higher collision energies and to investigate Rydberg electron transfer to other atoms with weakly bound negative ions (e.g., $\mathrm{Sr}$ and $\mathrm{Yb}$ ) to shed more light on the systematic changes of the electron transfer process with the binding energy. The case of $\mathrm{Yb}$ promises to be particularly interesting as a model system since the $\mathrm{Yb}$ atom is expected to have only one bound negative ion state with an electron affinity below $20 \mathrm{meV}$ [24]-[26].

This work has been supported by the Deutsche Forschungsgemeinschaft, by the Graduiertenkolleg "Laser- und Teilchenspektroskopie", by the Stiftung Rheinland-Pfalz für Innovation, by the European Union through the HCM network "Lasers, atoms and molecules: dynamical interactions" and by the U.S. National Science Foundation (Grant No. PHY-9509265). We thank E. LEBER for experimental support. We gratefully acknowledge M. MovRE and W. Meyer for helpful discussions and thank S. R. LeOne and T. Andersen for providing preprints of [14] and [8], respectively. 


\section{REFERENCES}

[1] Peterson J. R., Aust. J. Phys., 45 (1992) 293.

[2] Froese Fischer C., Lagowski J. B. and Vosko S. H., Phys. Rev. Lett., 59 (1987) 2263.

[3] Wijesundera W. P., Vosko S. H. and Parpia F. A., J. Phys. B, 29 (1996) 379.

[4] Salomonson S., Warston H. and Lindgren I., Phys. Rev. Lett., 76 (1996) 3092.

[5] Berkovits D., Boaretto E., Ghelberg S., Heber O. and Paul M., Phys. Rev. Lett., 75 (1995) 414.

[6] Petrunin V. V., Voldstad J. D., Balling P., Kristensen P. and Andersen T., Phys. Rev. Lett., 75 (1995) 1911.

[7] Petrunin V. V., Andersen H. H., Balling P. and Andersen T., Phys. Rev. Lett., 76 (1996) 744.

[8] Andersen H. H., Petrunin V. V., Kristensen P. and Andersen T., Phys. Rev. A, 55 (1997) 3247.

[9] Mclaughlin K. W. and Duquette D. W., Phys. Rev. Lett., 72 (1994) 1176.

[10] Fabrikant I. I., Phys. Rev. A, 48 (1993) R3411.

[11] Desfrançois C., Abdoul-Carime H. and Schermann J. P., Int. J. Mod. Phys. B, 10 (1996) 1339.

[12] Desfrançois C., Abdoul-Carime H., Adjouri C., Khelifa N. and Schermann J.-P., Europhys. Lett., 26 (1994) 25.

[13] Desfrançois C., Phys. Rev. A, 51 (1995) 3667.

[14] Lorensen H. Q., Parks H. V., Spain E. M., Smedley J. E., Greene C. H. and Leone S. R., Phys. Rev. A, 54 (1996) 1577.

[15] Khrebtukov D. B. and Fabrikant I. I., Phys. Rev. A, 54 (1996) 2906.

[16] Harth K., RaAb M., Ganz J., Siegel A., Ruf M.-W. and Ноtop H., Opt. Commun., 54 (1985) 343.

[17] Harth K., Ruf M.-W. and Нотор H., Z. Phys. D, 14 (1989) 149.

[18] Dunning F. B., J. Phys. Chem., 91 (1987) 2244; J. Phys. B, 28 (1995) 1645.

[19] Janev R. K., J. Chem. Phys., 64 (1976) 1891.

[20] Ruf M.-W., Yencha A. J., Hotop H., Movre M., Kerner C., Zillig S. and Meyer W., Z. Phys. D, 37 (1996) 219.

[21] Radtsig A. A. and Smirnov B. M., Sov. Phys. JeTP, 33 (1971) 282.

[22] Fabrikant I. I., J. Phys. B, 26 (1993) 2533.

[23] Haugen H. K., Andersen L. H., Andersen T., Balling P., Hertel N., Hvelplund P. and Möller S. P., Phys. Rev. A, 46 (1992) R1.

[24] Litherland A. E., Kilius L. R., Garwan M. A., Nadeau M.-J. and Zhao X.-L., J. Phys. B, 24 (1991) L233.

[25] Vosko S. H., Chevary J. A. and Mayer I. L., J. Phys. B, 24 (1991) 225.

[26] Andersen T., private communication. 